\title{
Assessment of psychiatric morbidity in patients attending a colposcopy clinic situated in a genitourinary medicine clinic
}

\author{
F C Boag, A M Dillon, J Catalan, R Edwards, S E Barton
}

\begin{abstract}
Objective-To determine the adverse psychological implications of referral for colposcopic screening within a genitourinary medicine clinic.
\end{abstract}

Design-Cross sectional survey.

Setting-A colposcopy clinic held within a genitourinary medicine clinic.

Subjects-160 consecutive women referred to the clinic were assessed for psychiatric morbidity using the General Health Questionnaire (GHQ-28). Women were referred to the colposcopy clinic because of one or more of the following: abnormal cervical cytology, condylomata acuminata, HIV antibody positivity. A history of prior local ablative therapy to the cervix was also included in the analysis.

Results-There was no difference in psychiatric morbidity, as detected by the GHQ-28, in women referred to the clinic because of abnormal cervical cytology or condylomata acuminata. The total GHQ-28 scores indicated a significant increase in psychiatric morbidity, with increased sub-scores indicating social dysfunction, anxiety and somatic symptoms, in women who had had prior laser therapy to the cervix. Women with HIV infection attending the clinic were noted to have an increase in GHQ-28 sub-scores indicating social dysfunction and depression.

Conclusions-Women who have undergone laser therapy to the cervix may benefit from psychological evaluation and supportive

\footnotetext{
John Hunter Clinic, 369 Fulham Road, London SW10 9TH

F C Boag, A M Dillon, S E Barton

Academic Department of Psychiatry, Prince of Wales Unit, Westminster Hospital, Dean Ryle Street, Horsferry Road, London SW18 2AP

Jose Catalan

Imperial Cancer Research Fund, Lincolns Inn Fields, London WC2A 3PX, UK

Robert Edwards
}

measures if they develop further genital lesions which require colposcopic evaluation. Women with HIV infection need further psychological evaluation prior to planning intervention and preventative strategies.

Introduction

The introduction of a screening programme into a population may induce fears and anxieties in persons selected for screening. ${ }^{1}$ Careful evaluation must be carried out to ensure that the benefits of the screening programme outweigh the problems which it causes. The number of women undergoing colposcopic examination of their lower genital tract continues to increase, whilst uncertainty persists about the criteria which should be used to identify those who will benefit most from this investigation. Within this debate most agree that colposcopy is valuable in the investigation of women whose cervical smear test shows dyskaryosis, but there is considerable disagreement as to whether women with particular conditions, such as genital wart virus infection or other sexually transmitted infections, should be offered colposcopic screening.

In evaluating such policies, reports of "false negative smear rates" and "unsuspected microinvasive disease" support increased referral for colposcopic screening, whilst surgical complications and restrictions on the availability of the service have acted as restraints. Little attention has been paid to the emotional and psychological implications for women selected for colposcopic screening.

This study set out to investigate women referred according to a variety of criteria who were attending a colposcopy clinic held in a genitourinary medicine clinic for evidence of psychiatric morbidity.

\section{Patients and methods}

All women making their first visit to a colposcopy clinic held within a genitourinary medicine clinic over an 11 month period were included in the study.

The criteria for referral to the colposcopy clinic were: abnormal cervical cytology, urethral, cervical, vaginal or persistent vulval condylomata acuminata, 
Table 1 Matrix depicting the number of patients attending the colposcopy clinic by referral criteria

\begin{tabular}{|c|c|c|c|c|c|c|}
\hline & & \multicolumn{4}{|c|}{ Patient group } & \multirow[b]{2}{*}{ Total } \\
\hline & & $\begin{array}{l}\text { Abnormal } \\
\text { cytology }\end{array}$ & $\begin{array}{l}\text { Condylomata } \\
\text { acuminata }\end{array}$ & $\begin{array}{l}\text { Prior } \\
\text { laser }\end{array}$ & $\begin{array}{l}\text { HIV } \\
\text { positive }\end{array}$ & \\
\hline $\begin{array}{l}\text { Patient } \\
\text { Group }\end{array}$ & $\begin{array}{l}\text { Abnormal cytology } \\
\text { Condylomata acuminata } \\
\text { Prior laser } \\
\text { HIV positive }\end{array}$ & $\begin{array}{r}42 \\
30 \\
6 \\
9\end{array}$ & $\begin{array}{r}30 \\
55 \\
9 \\
2\end{array}$ & $\begin{array}{l}6 \\
9 \\
4 \\
1\end{array}$ & $\begin{array}{l}9 \\
2 \\
1 \\
2\end{array}$ & $\begin{array}{l}86^{\star} \\
95^{\star} \\
19^{\star} \\
14\end{array}$ \\
\hline
\end{tabular}

*One patient had abnormal cytology, condylomata acuminata and prior laser.

or infection with HIV. In many of the women more than one of the referral criteria were present.

Women were referred to the colposcopy clinic after personal explanation of their results and the reason for colposcopic referral. They were encouraged to ask questions and to telephone the clinic if queries arose.

Each woman was given the General Health Questionnaire (GHQ-28), a self-administered questionnaire to complete immediately prior to her colposcopic examination. The questionnaire is a screening instrument which is known to be of value in establishing the likelihood of a patient having a psychiatric disorder. In addition to providing a total score, sub-scores for somatic symptoms, anxiety, social dysfunction and severe depression can be generated. The questionnaire is scored according to a standardised technique for psychiatric case finding. ${ }^{2}$

A full medical and gynaecological history, with particular reference to previous abnormal cervical smears and ablative or excisional cervical therapy was recorded.

Women were categorised into one or more of four groups according to the criteria for referral to the colposcopy clinic: (1) Abnormal cervical cytology. (2)
Urethral, cervical, vaginal or persistent vulval condylomata acuminata. (3) Previous laser therapy to the cervix followed by detection in our clinic of abnormal cytology or genital tract condylomata acuminata (as in group 2). (4) Known HIV infection. (table 1). For each of these groups the results of the total score and each sub-score of the GHQ-28 were analysed for any statistical significance using the Mann-Whitney U test.

\section{Results}

From August 1989 to June 1990, one hundred and sixty women completed the questionnaire. There were no refusals. Table 2 depicts the results of the $p$ values from the Mann-Whitney $U$ test for the difference in the GHQ-28 scores for each category, where the comparison is between all those in the group versus all those not in the group. There was a significant increase in total GHQ-28 score in patients who had prior laser therapy indicative of greater overall psychiatric morbidity $(p<0.001)$, and GHQ-28 sub-scores indicative of greater social dysfunction $(p=0.022)$, anxiety $(p<0.001)$ and somatic symptoms $(p<0.001)$.

Table 2 Summary statistics by group for the GHQ categories

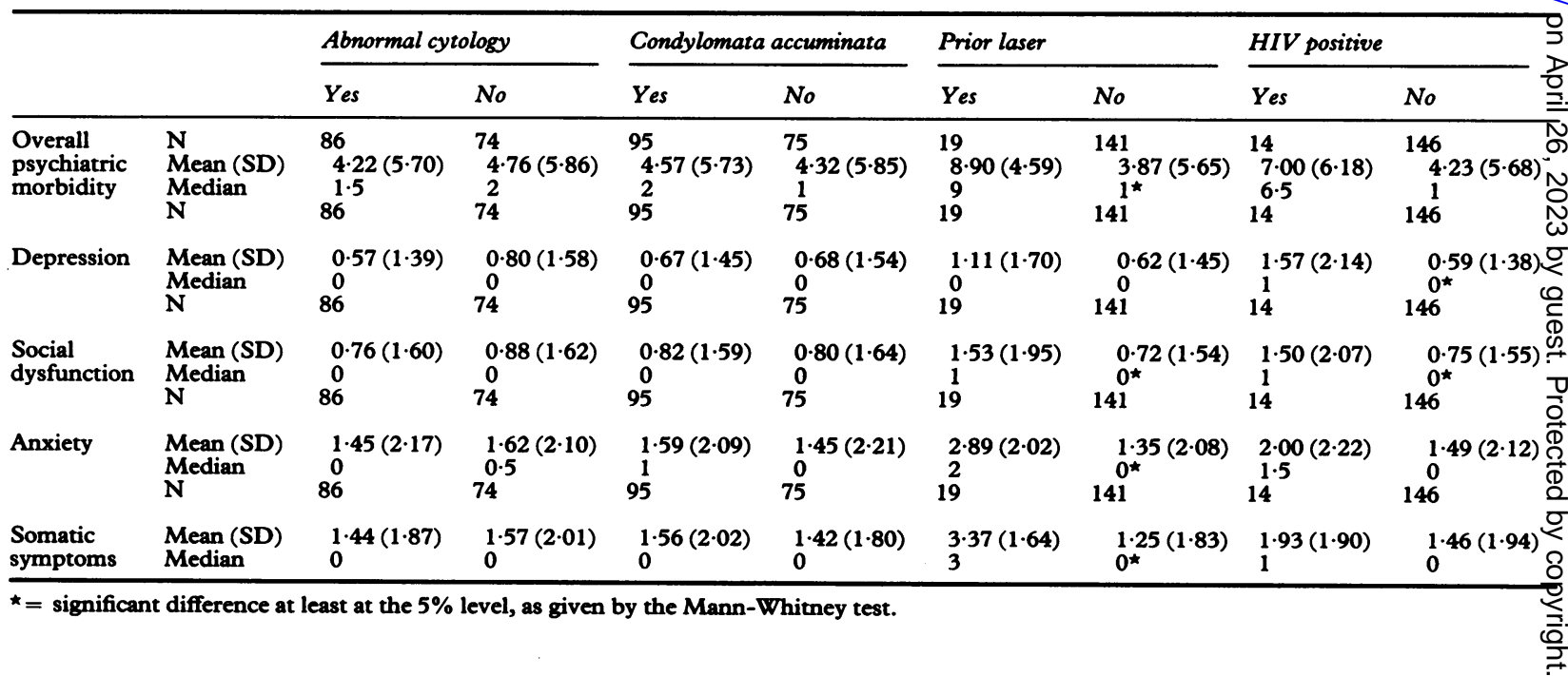


Women with HIV infection showed significantly increased GHQ-28 sub-scores indicating greater social dysfunction $(p=0.031)$ and depression $(p=0.004)$, although the small number of patients suggests caution in interpretation of the data.

There was no significant difference between women with abnormal cervical cytology or genital condylomata acuminata in overall psychiatric morbidity, depression, social dysfunction, anxiety or somatic symptoms as assessed by the GHQ-28.

A total of 86 women had abnormal cervical cytology; the GHQ-28 detected symptoms of abnormal anxiety in only eight.

We could attempt to model total GHQ-28 by four explanatory variables, the four group categories. Using GLIM, previous laser treatment was found to be a significant factor with $\chi^{2}=13.75, p=0.0002$. HIV seropositivity approaches significance with $\chi^{2}=$ $3.00, p=0.08$. Given previous treatment as a factor, HIV positivity is marginally significant, with $\chi^{2}=$ $3.91, \mathrm{p}=0.048$.

Overall, the linear modelling agrees with the results from the Mann-Whitney U test. Similar exercises could be undertaken with the four subcategories, depression, social dysfunction, anxiety and somative symptoms as dependent variables. However, as these variables only take values in the range $0-7$, this approach is not ideal. The limited range also reduces the power of the Mann-Whitney $\mathrm{U}$ test for these variables.

\section{Discussion}

This study demonstrates that psychiatric morbidity, detected by the GHQ-28, was present in a significant number of women who had previously undergone laser ablation of their cervix. This consisted of an increase in social dysfunction, anxiety and somatic symptoms. Previous workers have noted psychological abnormalities in women attending follow-up clinics after laser ablation, specifically the loss of attractiveness, low self esteem and anxiety, ${ }^{3}$ significant negative changes in sexual responsiveness ${ }^{4}$ and feelings of trauma, depression and vulnerability. ${ }^{5}$ Our study did not assess the duration of the changes; therefore it remains uncertain whether our findings detected pre-existing morbidity or morbidity induced by the new colposcopy referral. Correlational analysis of demographic factors was also not assessed in this study.

Some workers have observed a relationship between stress and the promotion of cervical cancer, ${ }^{6}$ and somatic anxiety and susceptibility to cervical cancer. ${ }^{7}$ Improvement of psychological well being of women with breast cancer has been claimed to prolong survival. ${ }^{8}$ We can therefore suggest that psychological evaluation and appropriate intervention may be beneficial to these patients for both physical and psychological reasons.
Significant depression and social dysfunction was detected by the GHQ-28 in the group of women with HIV infection. Further studies are required to assess whether this is due to HIV infection alone or the additional stress of a colposcopy referral. Such findings need to be incorporated in planning intervention strategies and/or reconsidering the referral criteria for these women, both inside and outside the context of research trials.

No differences in psychiatric morbidity were detected between patients referred for a colposcopy following an abnormal cervical smear or because of genital condylomata acuminata. This is consistent with a previous study in which sexual behaviour and attitudes to sex were assessed in women on their initial visit to a colposcopy clinic. No differences were detected between women referred by the following criteria: an abnormal cervical smear, partner of a male with penile papilloma virus infection or partner of a male with non-specific urethritis. ${ }^{4}$

In our study only eight of 86 women referred to the colposcopy clinic because of abnormal cytology had abnormal anxiety detected by the GHQ-28. This is in contrast to previous studies which have demonstrated high anxiety levels ${ }^{9}$ and anxiety, shock and anger $^{5}$ at initial colposcopy appointments. An explanation for this may be the method of referral. All our patients received a personal explanation of the reasons for referral, with adequate time for questions, on the day the appointment was arranged. Good explanation of abnormal cervical cytology results has been shown to reduce anxiety in patients requested to attend for a repeat cervical smear. ${ }^{10}$

In conclusion, this study demonstrates that women who have undergone previous laser therapy are psychologically more vulnerable than other women attending a new colposcopy referral. These women need further assessment to establish whether the morbidity was present prior to, or was induced by, the referral for colposcopy. Women who have undergone laser ablation need particular attention to be paid to psychological follow-up. If further referral to a colposcopy clinic is essential, specific counselling and psychiatric assessment should be carried out. The number of women with HIV infection who were assessed was small. However, psychological abnormalities were detected by the GHQ-28 and further investigation of these women is warranted. In addition, this study suggests that explanation, education and an open discussion at the time of referral to a colposcopy clinic may be beneficial and inclusion in colposcopic screening programmes would be advisable.

We thank Jane Hollihead for typing the manuscript and all the clinic nursing staff for their co-operation with this study.

Address and correspondence to Dr F C Boag 
1 Marteau TM. The psychological costs of screening. BMJ 1989;299:527.

2 Goldberg D. The General Health Questionnaire (GHQ-28). 1981 Nelson. Windsor.

3 McDonald TW, Neutens JJ, Fischer LM, Jesse D. Impact of cervical intraepithelial neoplasia diagnosis and treatment on self-esteem and body image. Gynecol Oncol 1989;34:345-9.

4 Campion MJ, Brown JR, McCance DJ, Atia W, Edwards R, Cuzick J, Singer A. Psychosexual trauma of an abnorma cervical smear. Br J Obstet Gynaecol 1988;95:175-81.

5 Posner T, Vessey M. Prevention of cervical cancer; The patients View 1988. London. King Edwards Hospital Fund.

6 Goodkin K, Antoni MH, Blaney PH. Stress and hopelessness in the promotion of cervical intraepithelial neoplasia to invasive squamous cell carcinoma of the cervix. $J$ Psychosom Res 1986;30:67-76

7 Antoni MH, Goodkin K. Host moderator variables in the promotion of cervical neoplasia. J Psychosom Res 1988;32: 327-38.

8 Spiegel D, Bloom JR, Kraemer HC, Gottheil E. Effect of psychosocial treatment on survival of patients with metastatic breast cancer. Lancet 1989;ii:888-91.

9 Marteau TM, Walker P, Giles J, Smail M. Anxieties in women undergoing colposcopy. Br JObstet Gynaecol 1990;97:859-61.

10 Wilkinson C, Jones JM, McBride J. Anxiety caused by abdominal result of cervical smear test: a controlled trial. $B M J$ 1990;300:440. 